$\mathrm{p}=0.042$ ], side effects [adjusted OR 2.23 95\% CI $(1.32$ to 3.74), $\mathrm{p}=0.002$ ] and lack of perception that the missed dose can aggravate the disease [adjusted OR 4.15, 95\% CI (1.03 to 16.67), $\mathrm{p}=0.045$ ] have emerged as determinants of the overall non-adherence.

Conclusion The results are in accordance with what has already been shown. The discrepancy observed between the two methods highlights the importance of access to biological methods. The identified risk factors will through regular evaluation, better identify PLWHA at greater risk of non-compliance and to offer enhanced compliance support.

\section{P3.24 LYMPHOGRANULOMA VENEREUM PROCTITIS ARE STILL INCREASING IN FRANCE}

'Bertille De Barbeyrac, ${ }^{2}$ Arabella Touati, ${ }^{2}$ Cécile Laurier-Nadalié, ${ }^{3}$ Chloé Le Roy, ${ }^{4}$ Nadège Hénin, ${ }^{4}$ Olivia Peuchant, ${ }^{5}$ Ndeindo Ndeikoundam, ${ }^{4}$ Cécile Bébéar, ${ }^{6}$ Guy La Ruche. 'University, USC Ea3671 Mycoplasmal and Chlamydial Infections in Humans, and National Reference Cente, Bordeaux, France; '2University, USC Ea3671 Mycoplasmal and Chlamydial Infections in Humans, and National Reference Cente, Bordeaux, France; ${ }^{3}$ University, USC Ea3671 Mycoplasmal and Chlamydial Infections in Humans, and National Reference Cente, Bordeaux, France; ${ }^{4}$ University, USC Ea3671 Mycoplasmal and Chlamydial Infections in Humans, and National Reference Cente, Bordeaux, France; ${ }^{5}$ French Institute for Public Health Surveillance, Saint Maurice, France; ${ }^{6}$ French Institute for Public Health Surveillance, Bordeaux, France

\subsection{6/sextrans-2017-053264.261}

Introduction Following the emergence of lymphogranuloma venereum (LGV) proctitis in the Netherlands in 2003, a voluntary surveillance system for LGV has been established in France. Based on the data of the National Reference Centre (NRC) for chlamydiae, Bordeaux, France, this study aimed to describe the epidemiology of LGV and non-LGV ano-rectal infection in France and to examine the characteristics of affected populations.

Methods The French surveillance network includes clinicians, biologists and NRC. Biologists sent rectal CT-positive samples to the NRC where the strains were typed by using two realtime PCR targeting $p m p \mathrm{H}$ gene specific of $\mathrm{L}$ and $\mathrm{L} 2 \mathrm{~b}$ strains. Biologists and clinicians performed a document on patient's clinical, biological and behavioural data. Clinical, biological and sexual risk behavioural variables were compared in men with LGV and with non-LGV cases according the HIV status using appropriate statistical tests over the period 2010-2015.

Results A total of 2627 LGV cases and 2633 non LGV cases were recorded from 2004 to 2015. In the period 2010 to 2015, there were 1747 LGV episodes in 1570 patients, most of whom were known to be HIV-positive (841/1105, 76.1\%) and 2242 non LGV episodes in 2051 patients, most of whom were known to be HIV-negative (1186/1665, 71.2\%). The number of LGV diagnoses was multiplied by 2.4 between 2012 and 2015 and the number of recurrence reached 10\% in 2015. LGV continues to affect a core group: HIV-infected MSM who engage in high-risk sexual practices. They were older and more often infected with syphilis than men with non-LGV cases, were usually symptomatic and mainly lived in Paris. Those who acquired LGV reinfection had concurrent hepatitis $\mathrm{C}$ and syphilis more often than those with a single episode.

Conclusion A steady annual increase in the number of LGV cases and in the number of LGV recurrences was observed since 2012, demonstrating that the LGV epidemic is not under control and requires providing better information about the disease to affected patients and physicians of all specialties who take care them

\section{P3.25 QUANTIFICATION OF THE RISK OF PELVIC INFLAMMATORY DISEASE FOLLOWING A CHLAMYDIA TRACHOMATIS TEST BY DIAGNOSTIC TEST TYPE}

${ }^{1}$ Bethan Davies, ${ }^{2}$ Katy Me Turner, ${ }^{3}$ Berit Andersen, ${ }^{4}$ Henrik Westh, ${ }^{1}$ Helen Ward. ${ }^{1} /$ mperial College London, London - Great Britain; ${ }^{2}$ University of Bristol, Bristol - Great Britain; ${ }^{3}$ Department of Public Health Programmes, Randers - Denmark; ${ }^{4}$ Hvidovre University Hospital, Copenhagen - Denmark

\subsection{6/sextrans-2017-053264.262}

Introduction Nucleic Acid Amplification Tests (NAATs) are the recommended test type for diagnosing Chlamydia trachomatis (chlamydia). However poorer performing methods remain in use. We compared the risk of pelvic inflammatory disease (PID) in women tested for chlamydia by diagnostic test type.

Methods We used a sub-set of the Danish Chlamydia study that included all female residents of Denmark who were tested for chlamydia (1998-2001) when aged 15-34 years. Chlamydia tests performed on urinary or genital samples with a definitive positive or negative result were categorised as non-NAAT (ELISA; IF; “antigen") or NAAT (PCR; SDA; TMA; LCR; DNA/RNA) and limited to each woman's first test. Test records were linked to hospital presentations for PID within 12 months. Women with previous PID or PID diagnosed on the same date as the test were excluded. We used logistic regression to compare the risk of PID by test type adjusted for age, test year and test result.

Results Of the 272105 women in the study, $44.78 \%$ were tested using NAAT, $6.38 \%$ tested positive for chlamydia and $0.64 \%$ were diagnosed with PID within 12 months. Overall, the adjusted risk of PID within 12 months of a chlamydia test was higher following a positive test (AOR 1.40 (95\% CI 1.18$1.67)$ and in older women (25-34 years $1.36(1.23-1.49)$ ) and lower in women tested using a NAAT $(0.87(0.78-0.96))$ and in the more recent time interval (2000/2001 $0.89(0.80-$ 0.99)). In women with a positive test, and presumably treated infection, the risk of PID did not differ by test type (1.25 (0.87-1.79)). In women with a negative test, the risk of PID was lower following a NAAT $(0.84(0.75-0.93))$.

Conclusion Women with a negative result from a non-NAAT chlamydia test have a $16 \%$ higher risk of PID by 12 months compared to women with a negative result from a NAAT. This is presumably due to the increased proportion of false negative tests with the less sensitive non-NAATs. This study quantifies the health impact of using poorer performing chlamydia diagnostic tests and provides further evidence for phasing them out.

\section{P3.26 THE EFFICACY OF AZITHROMYCIN AND DOXYCYCLINE TREATMENT FOR RECTAL CHLAMYDIAL INFECTION: A RETROSPECTIVE COHORT STUDY IN SOUTH AUSTRALIA}

$\mathrm{B} \mathrm{Li}{ }^{1,2},{ }^{3} \mathrm{JS}$ Hocking, P Bi ${ }^{1}, \mathrm{C}$ Bell ${ }^{2}$, A Ward ${ }^{2}, \mathrm{CK}$ Fairley ${ }^{4,5} .{ }^{1}$ The University of Adelaide, Australia; ${ }^{2}$ Royal Adelaide Hospital, Australia; ${ }^{3}$ University of Melbourne, Australia; ${ }^{4}$ Monash University, Australia; ${ }^{5}$ Melbourne Sexual Health Centre, Australia

\subsection{6/sextrans-2017-053264.263}

Introduction British Association for Sexual Health and HIV (BASHH) and Australian guidelines recommend either 